\title{
Contents
}

Special Issue on Experimental Aspects of Quantum Computing $\ldots \ldots \ldots \ldots \ldots \ldots$ Henry Everitt

Invited Articles

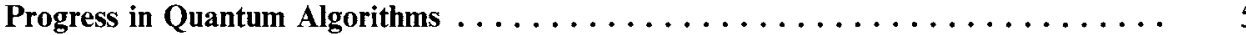
Peter W. Shor

NMR Quantum Information Processing. . . . . . . . . . . . . . . . . 15

Chandrasekhar Ramanathan, Nicolas Boulant, Zhiying Chen, David G. Cory, Isaac Chuang, and Matthias Steffen

Quantum Computing with Trapped Ion Hyperfine Qubits. . . . . . . . . . .

B. B. Blinov, D. Leibfried, C. Monroe, and D. J. Wineland

Ion Trap Quantum Computing with $\mathbf{C a}^{+}$Ions $\ldots \ldots \ldots \ldots \ldots \ldots$

R. Blatt, H. Häffner, C. F. Roos, C. Becher, and F. Schmidt-Kaler

Quantum Information Processing in Cavity-QED

S. J. van Enk, H.J. Kimble, and H. Mabuchi

Quantum Information Processing with Trapped Neutral Atoms.

P. S. Jessen, I. H. Deutsch, and R. Stock

The Road to a Silicon Quantum Computer. . . . . . . . . . . . . . .

$J$. R. Tucker and T.-C. Shen

Controlling Spin Qubits in Quantum Dots

Hans-Andreas Engel, L. P. Kouwenhoven, Daniel Loss, and C. M. Marcus

Spin-based Quantum Dot Quantum Computing in Silicon . . . . . . . . . . . .

Mark A. Eriksson, Mark Friesen, Susan N. Coppersmith, Robert Joynt, Levente

J. Klein, Keith Slinker, Charles Tahan, P. M. Mooney, J. O. Chu, and S. J.

Koester

Optically Driven Quantum Computing Devices Based on Semiconductor Quantum Dots Xiaoqin Li, Duncan Steel, Daniel Gammon, and L. J. Sham

Implementing Qubits with Superconducting Integrated Circuits.

Michel H. Devoret and John M. Martinis

Towards Scalable Linear-Optical Quantum Computers . . . . . . . . . . . . .

J. P. Dowling, J. D. Franson, H. Lee, and G. J. Milburn

Photonic Technologies for Quantum Information Processing . . . . . . . . . . .

Prem Kumar, Paul Kwiat, Alan Migdall, Sae Woo Nam, Jelena Vuckovic, and

Franco N. C. Wong 


\section{Contributed Articles}

Quantum Computer Development with Single Ion Implantation. . . . . . . . . . . 233

A. Persaud, S. J. Park, J. A. Liddle, I. W. Rangelow, J. Bokor, R. Keller, F. I. Allen, D. H. Schneider, and T. Schenkel

Bang-Bang Refocusing of a Qubit Exposed to Telegraph Noise . . . . . . . . . . . 247

Henryk Gutmann, Frank K. Wilhelm, William M. Kaminsky, and Seth Lloyd

Quantum Computing and Information Extraction for Dynamical Quantum Systems . . 273 Giuliano Benenti, Giulio Casati, and Simone Montangero

One-Dimensional Continuous-Time Quantum Walks . . . . . . . . . . . . . . . . . 295

D. ben-Avraham, E. M. Bollt, and C. Tamon 